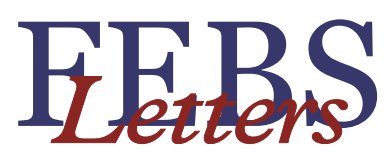

journal homepage: www.FEBSLetters.org

\title{
ANKHD1 regulates cell cycle progression and proliferation in multiple myeloma cells
}

\author{
Anamika Dhyani ${ }^{\mathrm{a}, *}$, Adriana S.S. Duarte ${ }^{\mathrm{a}}$, João A. Machado-Neto ${ }^{\mathrm{a}}$, Patricia Favaro ${ }^{\mathrm{a}, \mathrm{b}}$, \\ Manoela Marques Ortega ${ }^{a}$, Sara T. Olalla Saad ${ }^{a}$ \\ ${ }^{a}$ Hematology and Hemotherapy Center, University of Campinas/Hemocentro-Unicamp, Instituto Nacional de Ciência e Tecnologia do Sangue, Campinas, São Paulo, Brazil \\ ${ }^{\mathrm{b}}$ Department of Biological Sciences, Federal University of São Paulo, Diadema, São Paulo, Brazil
}

\section{A R T I C L E I N F O}

\section{Article history:}

Received 8 August 2012

Revised 11 October 2012

Accepted 23 October 2012

Available online 6 November 2012

Edited by Angel Nebreda

\section{Keywords:}

ANKHD1

Multiple myeloma

Proliferation

Cell cycle

p21

\begin{abstract}
A B S T R A C T
ANKHD1 is a multiple ankyrin repeat containing protein, highly expressed in cancers, such as acute leukemia. The present study was undertaken to determine the expression and functional significance of ANKHD1 in human Multiple Myeloma (MM). We found that ANKHD1 is highly expressed in MM patient cells and cell lines. In vitro, lentiviral mediated ANKHD1-shRNA inhibited proliferation and delayed $S$ to G2M cell cycle progression in glucocorticoid resistant (U266) and sensitive (MM1S) MM cells. Further ANKHD1 silencing resulted in upregulation of cyclin dependent kinase inhibitor p21 irrespective of the p53 status of the MM cell lines. These data suggest that ANKHD1 might have a role in MM cell proliferation and cell cycle progression by regulating expression of p21. (C) 2012 Federation of European Biochemical Societies. Published by Elsevier B.V. All rights reserved.
\end{abstract}

\section{Introduction}

Multiple myeloma (MM) is a hematologic malignancy characterized by the clonal proliferation of malignant plasma cells in the bone marrow [1-3]. Development of MM is a multistep process often associated with an increasing frequency of chromosomal abnormalities and complex translocations that lead to overexpression of known and putative oncogenes [4,5]. Current therapies for the disease include chemotherapy with or without stem cell transplantation, glucocorticosteroids, thalidomide, the proteosome inhibitor bortezomib, and combinations of these agents [6-8]. However, most of these treatments are not curative and therefore development of new treatment approaches is required for effective anti-cancer therapeutics. One major step towards the development of cancer therapeutics is the functional characterization of actively expressed genes which could possibly be targeted for cancer treatment [9-13]. ANKHD1, Ankyrin repeat and KH domain containing 1 , is one such gene overexpressed by primary acute leukemia cells and leukemia cell lines [14]. ANKHD1 is located on human chromosome $5 q 31.3$ as a single copy and encodes for a protein with multi-

Abbreviation: ANKHD1, Ankyrin repeat and KH domain containing 1

* Corresponding author. Address: Hematology and Hemotherapy Center, University of Campinas, Rua Carlos Chagas, 480, CEP 13083-878, Campinas, SP, Brazil. Fax: +551932891089.

E-mail address: anamikadhyani2009@gmail.com (A. Dhyani). ple ankyrin repeats and as single KH domain (ANKHD1). ANKHD1 is thought to function as a scaffolding protein associated with abnormal phenotype of leukemia cells. Earlier study reports association of this protein with $\mathrm{SH} 2$-containing protein-tyrosine phosphatase (SHP2) in human leukemia cell line K562 and prostate cancer cell line LNCaP, suggesting it to be an adaptor protein required for leukemogenesis and development of cancer [14]. Till date, little is known regarding ANKHD1 and hence, its role in the development of cancer and cancer therapeutics warrants investigation [14].

Owing to the ubiquitous expression of ANKHD1 and probable role in the development of cancer, we set out to investigate ANKHD1 expression and role in MM. In this study, we report for the first time the functional role of ANKHD1 in the proliferation of MM cells. We found that ANKHD1 promotes proliferation by regulating the cell cycle progression by downregulating p21 expression.

\section{Materials and methods}

\subsection{Primary cells and cell culture}

Primary tumor cells from bone marrow aspirates of MM patients $(n=10)$ were purified by Ficoll Hypaque separation (GE healthcare), followed by $\mathrm{CD} 138^{+}$microbead selection (Miltenyi Biotech, Auburn, CA). Four of ten MM patients (40\%) patients had 
the immunoglobulin G myeloma type (IgG), three (30\%) had the immunoglobulin A myeloma type (IgA), two (20\%) had light-chain MM (kappa) and for one MM patient immunoglobulin type was not available. $\mathrm{CD} 138^{+}$(plasma) cell purity was above 95\% as determined by flow cytometry. Purified plasma cells from bone marrow mononuclear cells $(n=2)$ and tonsils $(n=4)$ of healthy donors were used as controls. All healthy donors and patients provided informed written consent and the study was approved by the ethics committee of the University of Campinas.

Human myeloma cell lines MM1R, MM1S, RPMI 8226 and U266 were a kind gift from Prof. Lionel J. Coignet, Department of Cancer Genetics, Roswell Park Cancer Institute, Buffalo, New York, USA. MM cell lines were cultured in RPMI-1640 medium supplemented with $10 \%$ heat inactivated Fetal calf serum (FCS), glutamine, $100 \mu \mathrm{g} / \mathrm{ml}$ Penicillin and $100 \mu \mathrm{g} / \mathrm{ml}$ Streptomycin. 293T cells for viral transfection were grown in DMEM containing 10\% FCS. HT1080 cells used for viral titration were grown in complete MEM medium. All cells were maintained at $5 \% \mathrm{CO}_{2}$ and $37{ }^{\circ} \mathrm{C}$ temperature in $\mathrm{CO}_{2}$ incubator.

\subsection{Quantitative Real Time-PCR ( $q R T-P C R)$}

Total RNA was extracted from cells using the TRIzol reagent, according to the manufacturer's instructions (Invitrogen, Carlsbad, CA, USA). The reverse transcription reaction was performed using RevertAid $^{\mathrm{TM}}$ First Strand cDNA Synthesis Kit, according to the manufacturer's instructions (MBI Fermentas, St. Leon-Rot, Germany). Expression of ANKHD1 mRNA was detected by Maxima Sybr green qPCR master mix, according to the manufacturer's instructions (MBI Fermentas, St. Leon-Rot, Germany) in the ABI 7500 Sequence Detection System (PE Applied-Biosystem) using specific primers: forward 5'-TGTCCGAGGTTGAATCATTTT-3', reverse 5'-GTCCGAGG TTGAATCATTTTTT-3'; HPRT was used as endogenous control. The forward and reverse primers for HPRT were 5'-GAACGTCTTGCTCGA GATGTGA-3' and 5'-TCCAGCAGGTCAGCAAAGAAT-3, respectively. Relative levels of gene expression were quantified using the equation, $2^{-\Delta \Delta C T}$ [15]. A negative 'no template control' was included for each primer pair. The dissociation protocol was carried out at the end of each run to check for non-specific amplification. Three replicas were run on the same plate for each sample.

\subsection{Western blot}

MM cell lines were harvested when in exponential phase and used for extraction of proteins. Briefly, cells were lysed in ice-cold buffer (100 mM Tris- $\mathrm{HCl} \mathrm{pH}$ 7.5, $10 \mathrm{mM}$ EDTA, 10\% Triton X, $100 \mathrm{mM} \mathrm{NaF}$ and phosphatase and protease inhibitors). Protein content of the extracts was determined by Bradford assay. Equal amounts of total protein extracts were then subjected to SDSPAGE followed by Western blot analysis with the indicated antibodies and ECL ${ }^{\mathrm{TM}}$ Western Blotting Analysis System (Amersham Pharmacia Biotech, UK Ltd., Buckinghamshire, England) as described [15]. Polyclonal antibodies against ANKHD1, actin, cdk2, cdk4 and p27 were from Santa Cruz Biotechnology (Santa Cruz, CA, USA). Monoclonal antibody against p21 and p53 were from Cell Signaling Technologies (Cell Signaling, Danvers, MA, USA).

\subsection{Laser confocal analysis}

MM cell lines were fixed in $4 \%(\mathrm{w} / \mathrm{v})$ paraformaldehyde in PBS (pH 7.4) for $30 \mathrm{~min}$ at $25^{\circ} \mathrm{C}$ and washed two times for $5 \mathrm{~min}$ each with PBS containing $0.5 \%(\mathrm{w} / \mathrm{v})$ glycine. The washed cells were allowed to adhere on $0.01 \%(\mathrm{w} / \mathrm{v})$ poly-L-lysine coated coverslips, permeabilized with $0.2 \%(\mathrm{v} / \mathrm{v})$ Triton X-100 (5-10 min) and blocked with $1 \%$ BSA in PBS for 30 min. Cells were incubated overnight at $4{ }^{\circ} \mathrm{C}$ with primary antibody against ANKHD1 (1:200 dilution). After washing, the slides were labelled with Alexa Fluor 633-conjugated anti-goat antibody (Molecular Probes, Leiden, Netherlands) for $2 \mathrm{~h}$. Cells were then incubated with FITC-conjugated phalloidin (Sigma-Aldrich, MO, USA) at room temperature and coverslips with ProLong Gold antifade reagent with DAPI (Molecular Probes, Leiden, Netherlands) were applied. Positive immunoreactivity was visualized by laser confocal scanning (Zeiss LM510). In the absence of primary antibodies, application of secondary antibodies (negative controls) failed to produce any significant staining.

\subsection{Lentivrus production and transduction of MM cell lines}

Two short hairpin RNAs (shRNAs) targeting ANKHD1 gene were designed and cloned in lentivirus expressing vectors as per manufacturers protocol (BLOCK-iT ${ }^{\mathrm{TM}}$ Lentiviral RNAi system, Invitrogen). The RNAi candidate target sequences for ANKHD1 were 5'TGTCCGAGGTTGAATCATTTT-3' (ANKHD1 shRNA1) and 5'-ATGCACACTGCCTTAATGTT-3' (ANKHD1 shRNA2). shRNA expressing viruses were produced in $293 \mathrm{~T}$ cells according to standard protocols. Lentiviral shRNA vector against LacZ gene supplied by Invitrogen was used as the negative control.

MM cell lines were transduced with lentiviral ANKHD1 shRNA vectors or control vector (Control shRNA) in the presence of $4 \mu \mathrm{g}$ / $\mathrm{ml}$ polybrene (Sigma-Aldrich, St. Louis, MO, USA) at multiplicity of infection (MOI) of 1 and centrifuged at $800 \times g$ for $1 \mathrm{~h}$. Transduced cells were then subjected to blasticidin selection for 10-15 days. Blasticidin resistant cells were expanded and analyzed for proliferation, apoptosis and cell cycle or lysed for Western blotting.

\subsection{Methylthiazoletetrazolium (MTT) assay for cell proliferation}

Control shRNA and ANKHD1 shRNA cells were serum-starved in $0.5 \% \mathrm{FBS}$ for $12 \mathrm{~h}$. A total of $5 \times 10^{4}$ cells per well were then plated in a 96-well plate in RPMI $10 \%$ FBS and incubated in a $\mathrm{CO}_{2}$ incubator for 24,48 and $72 \mathrm{~h}$ at $37^{\circ} \mathrm{C}$. After incubation, $5 \mathrm{mg} / \mathrm{ml}$ solution of MTT was added to the wells and incubated at $37^{\circ} \mathrm{C}$ for $4 \mathrm{~h}$. The reaction was interrupted by using $0.1 \mathrm{~N} \mathrm{HCl}$ in anhydrous isopropanol. Cell growth was evaluated by measuring the absorbance at $570 \mathrm{~nm}$, using an automated plate reader. All conditions were tested in six replicates.

\subsection{Cell cycle analysis by flow cytometry}

Cells were fixed in $70 \%$ ethanol, for at least $2 \mathrm{~h}$ at $4{ }^{\circ} \mathrm{C}$, and stained with $20 \mu \mathrm{g} / \mathrm{ml}$ propidium iodide (PI) containing $10 \mu \mathrm{g} / \mathrm{ml}$ RNase A for $30 \mathrm{~min}$ at room temperature. Fluorescence cell analysis was performed with a FACSCalibur (Becton-Dickinson, CA, USA). Resulting DNA distributions were analyzed by Modifit (Verify Software House Inc., Topsham, ME, USA) for the proportions of cells in the phases of the cell cycle.

\subsection{Assessment of apoptosis by Annexin-V and PI staining}

Control shRNA and ANKHD1 shRNA cells were seeded on 12well plates for $48 \mathrm{~h}$. Cells were then harvested at 24 and $48 \mathrm{~h}$, washed twice with ice cold PBS and resuspended in binding buffer containing $1 \mu \mathrm{g} / \mathrm{ml}$ PI and $1 \mu \mathrm{g} / \mathrm{ml}$ FITC labeled Annexin-V. All specimens were analyzed on a FACS Calibur after incubation for $15 \mathrm{~min}$ at room temperature in a light-protected area. Ten thousand events were acquired for each sample.

\subsection{Statistical analysis}

Statistical analysis was performed using GraphPad Instat 5 (Graph-Pad Software, Inc., San. Diego, CA, USA). For comparisons, an appropriate Student's $t$-test or Mann-Whitney test was used. 
Values of ${ }^{*} P<0.05$ were considered as statistically significant. All experiments were repeated at least three times.

\section{Results}

\subsection{ANKHD1 expression and localization}

We used qRT-PCR to evaluate the expression of ANKHD1 in plasma cells from MM patients and compared with that from healthy donors. We found that plasma cells from MM patients had higher expression of ANKHD1 when compared with plasma cells from healthy donors $\left({ }^{*} P=0.004\right)$ (Fig. $\left.1 \mathrm{a}\right)$.

We also analyzed the expression of ANKHD1 mRNA in four MM cell lines namely MM1R, MM1S, RPMI 8226 and U266. qRT-PCR showed abundant expression of ANKHD1 mRNA in MM1S and MM1R cell lines followed by RPMI 8226 and U266 cell lines (Fig. 1b). In all four cell lines the expression was higher (2-5-fold) than the positive control (K562). These findings were further corroborated by Western blot analysis of ANKHD1 expression in these cell lines, standardized to actin expression (Fig. 2a), which showed high levels of ANKHD1 expression in MM1S and MM1R cell lines, markedly lower levels in U266 cells. However RPMI 8226 that had lower mRNA expression as compared to MM1S and MM1R cells, showed the highest expression in Western blot. The expression of ANKHD1 was characterized with a thick band of approximately $270 \mathrm{kDa}$ in the immunoblot of MM cells, and expression of ANKHD1 in K562 was used as a positive control, as previously described.

Laser confocal analysis of all four MM cell lines studied showed that ANKHD1 is predominantly located in the cytoplasm (Fig. 2b).
3.2. Specific inhibition of ANKHD1 mRNA and protein expression by ANKHD1 specific shRNA

To understand the significance of ANKHD1 upregulation in MM patients and cell lines, we used lentivirus vectors expressing ANKHD1 specific shRNA for gene silencing. MM cell line, U266 was transduced with ANKHD1 shRNA1 and 2, separately. Degree of knockdown in ANKHD1 expression was determined by qRTPCR analysis and Western blot. As shown in Fig. 3a and 3b, both ANKHD1 specific shRNAs substantially decreased $\left({ }^{*} P<0.05\right)$ ANKHD1 mRNA and protein expression in U266 cells. However of the two, ANKHD1 shRNA1 showed higher efficiency with 70\% and $80 \%$ inhibition in endogenous ANKHD1 mRNA and protein as compared to $45 \%$ and $70 \%$ in case of ANKHD1 shRNA2 (Fig 3a and b). MM1S transduced with ANKHD1 shRNA1 also showed significant $\left({ }^{*} P<0.05\right)$ downregulation of ANKHD1 expression with $44 \%$ and $50 \%$ inhibition of endogenous ANKHD1 mRNA and protein, respectively. The effect of ANKHD1 silencing was specific, as cells transduced with control shRNA (Lac $Z$ gene) failed to knockdown ANKHD1 expression (Fig. 3). Also, there was no significant difference in ANKHD1 expression between untransduced and negative control shRNA vector transduced cells (data not shown).

\subsection{Effect of ANKHD1 knockdown on proliferation and apoptosis}

To determine whether silencing ANKHD1 by shRNA had an inhibitory effect on MM cells growth, cell proliferation was determined by MTT assay. Fig. 4 shows that inhibition of ANKHD1 expression by shRNA suppressed proliferation of MM cells.

A
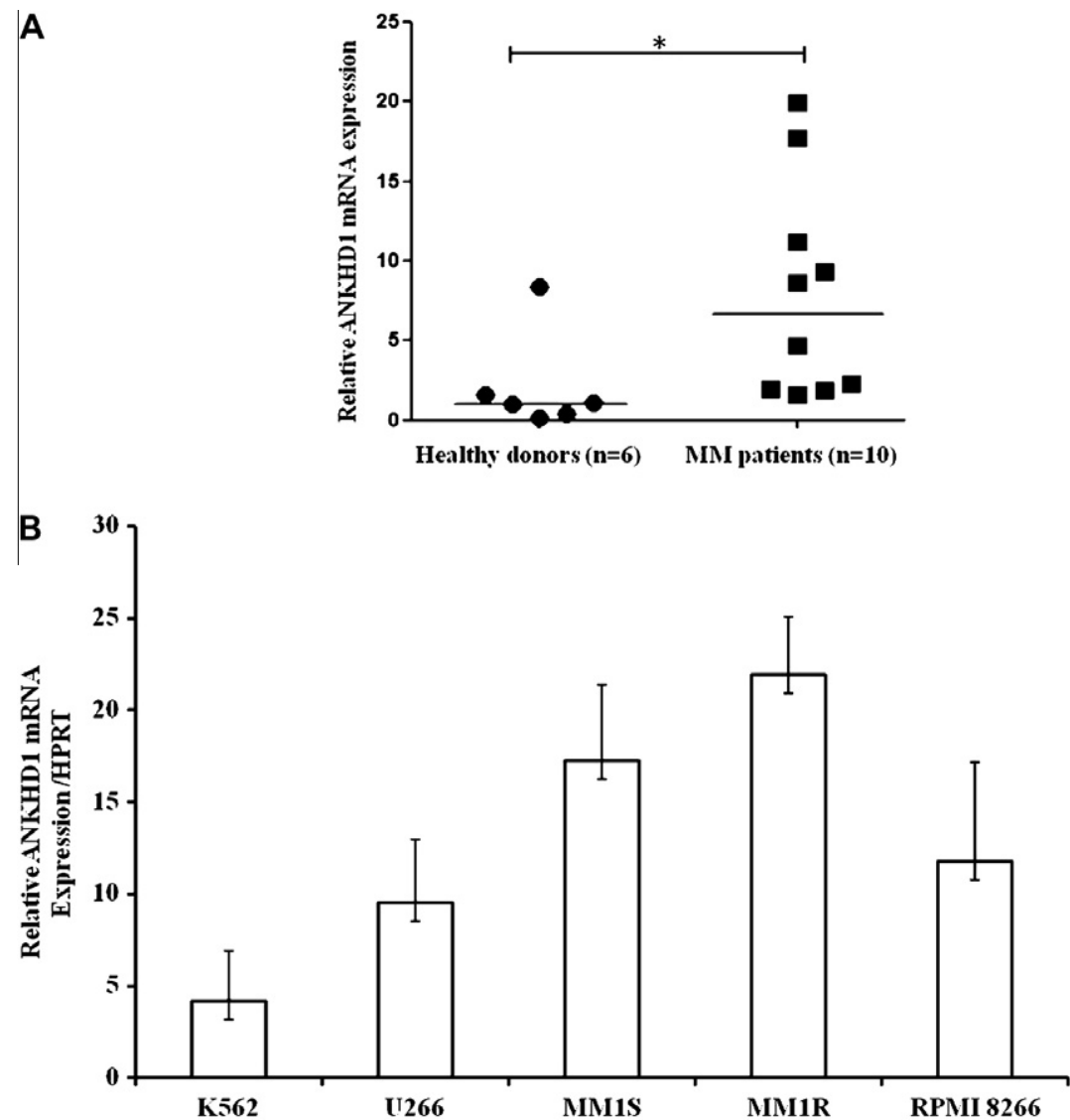

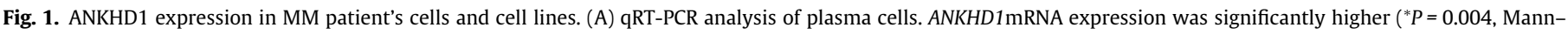

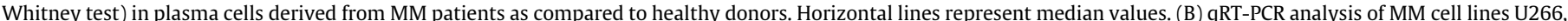

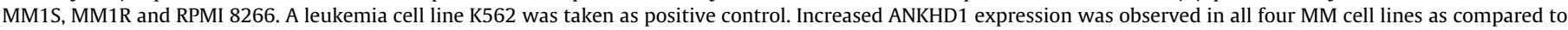
positive control (K562). 

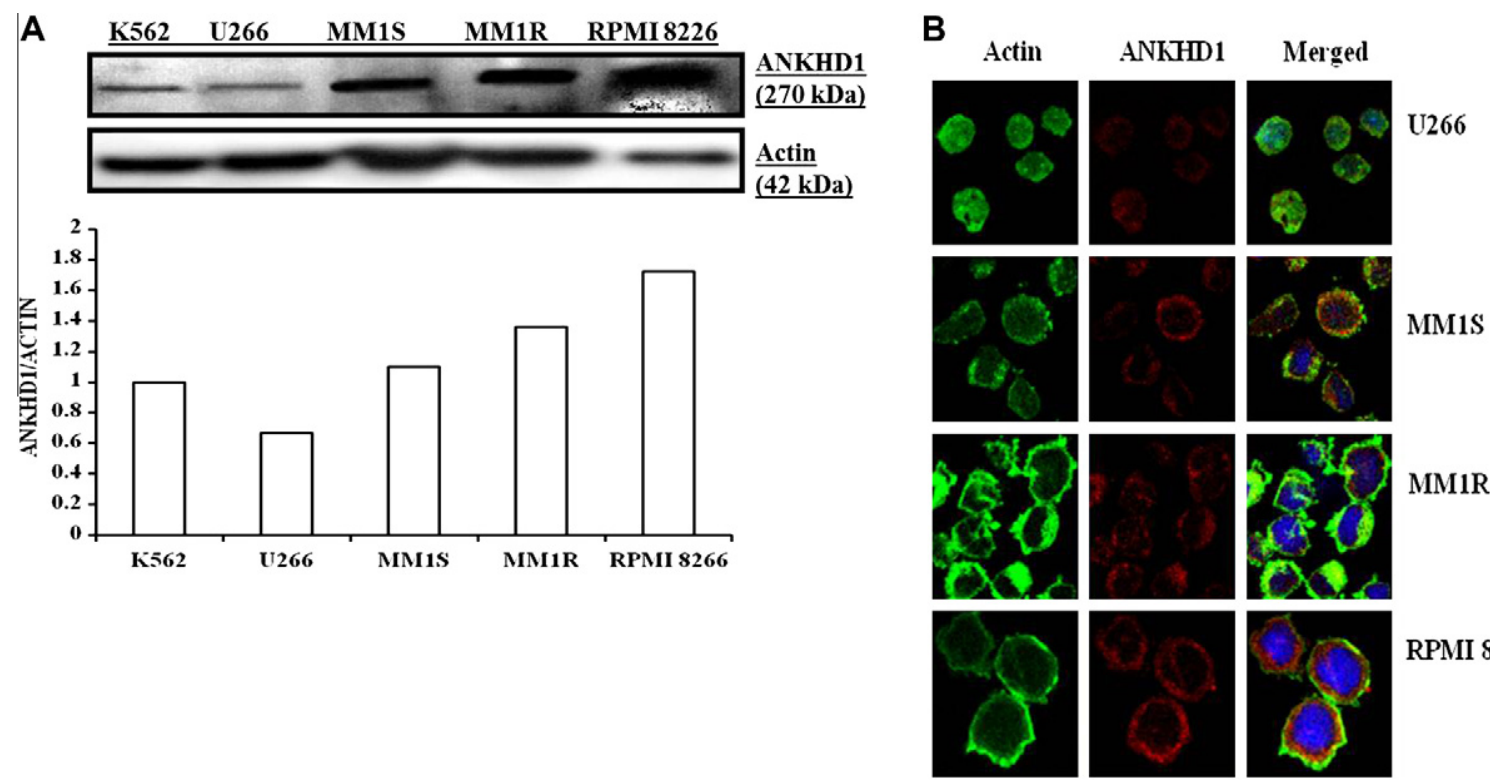

MMIR

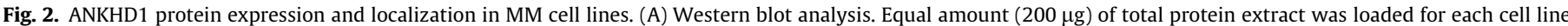

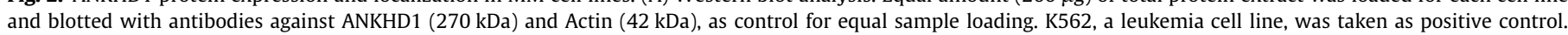

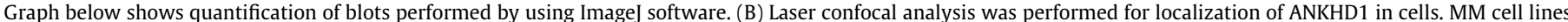

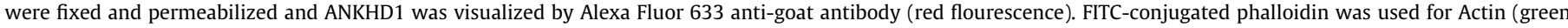

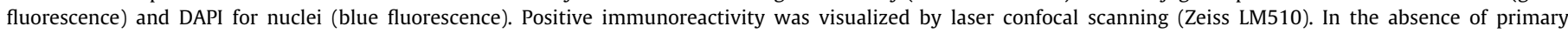

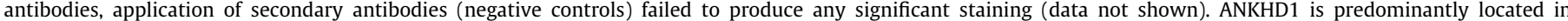
cytoplasm of all four MM cell lines.
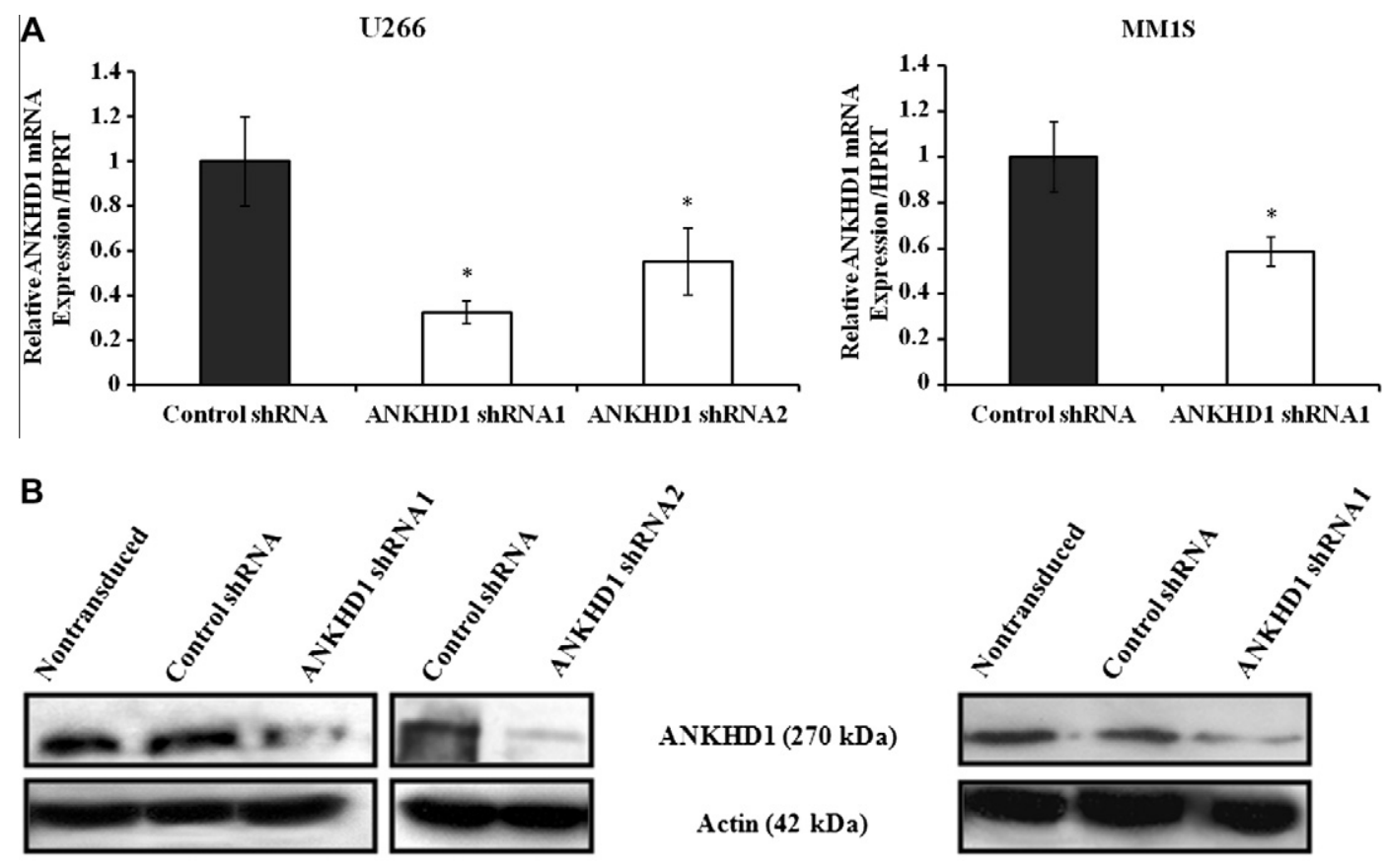

ANKHDl $(270 \mathrm{kDa})$

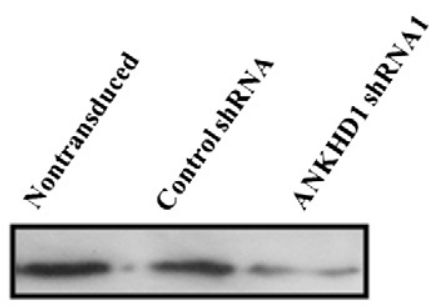

Actin (42 kDa)

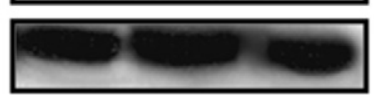

Fig. 3. Inhibition of ANKHD1 expression by specific shRNA. (A) Quantitative expression of ANKHD1 mRNA in ANKHD1 inhibited (ANKHD1 shRNA1, 2 or both) and control (control shRNA) cells. Lentivirus-mediated ANKHD1 shRNA effectively silenced ANKHD1 in MM cell lines (U266 \& MM1S), ${ }^{*} P<0.05$, Student's $t$-test. Both the ANKHD1 specific shRNAs (ANKHD1 shRNA1 and ANKHD1 shRNA2) effectively inhibited ANKHD1 mRNA expression in U266 cells. mRNA expression levels were normalized by HPRT as endogenous control. Results were analyzed using $2^{-\Delta \Delta C T}$. Experiments were performed in triplicates. (B) Western blot analysis of non-transduced, control shRNA and ANKHD1 shRNA total cell extracts (U266 \& MM1S). The membranes were blotted with antibodies against ANKHD1 or Actin (as a control for equal sample loading), and developed with the ECL Western blotting analysis system.

Proliferation was significantly reduced by $70 \%$ (U266) and $44 \%$ (MM1S) in ANKHD1 shRNA1 knockdown cells when compared with control cells $\left({ }^{*} P<0.05\right)$ at $48 \mathrm{~h}$ after incubation. Significant inhibition of proliferation was also observed in U266 cells transduced with ANKHD1 shRNA2. There was significant difference $\left({ }^{*} P<0.05\right)$ in proliferation between control and ANKHD1 knockdown MM cells at all three time points studied (Supplementary Fig. 1).

We further evaluated the effect of inhibiting ANKHD1 expression by shRNA on apoptosis in MM cells. However, annexin-V anal- 
A

U266

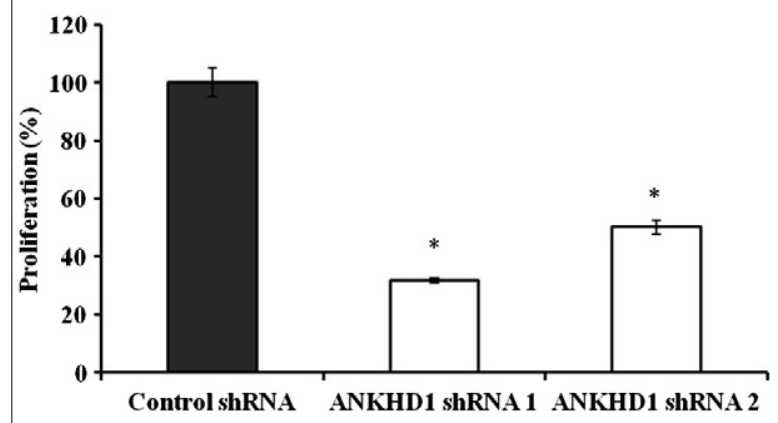

B

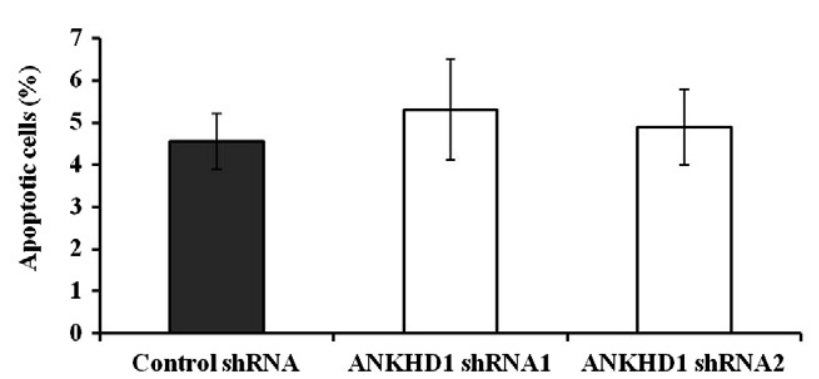

MM1S

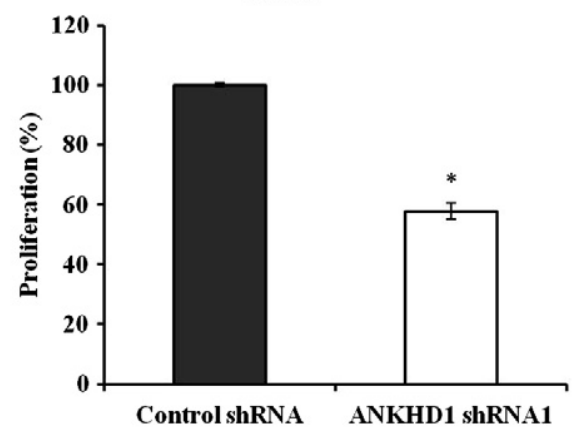

MM1S

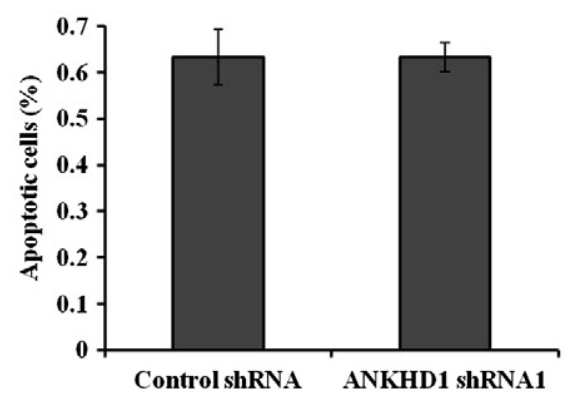

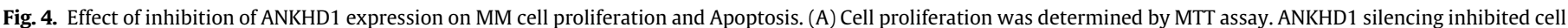

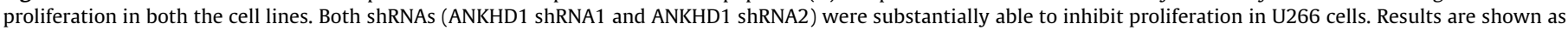

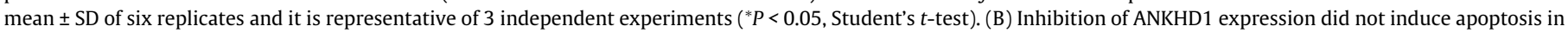
MM cell lines. Apoptosis in cells was determined by Annexin-V and PI staining.

ysis showed no significant increase in apoptosis of cells transduced with either of ANKHD1 shRNAs (Fig. 4b). This, therefore, indicates that knockdown of ANKHD1 expression by shRNAs is not significantly effective in inducing apoptosis in MM1S and U266 cells. Similar effects on proliferation and apoptosis, observed after ANKHD1 silencing with both the shRNAs, implies that the effects on cell functions are the result of RNAi-mediated silencing of the targeted gene rather than off-target effects. Since ANKHD1 shRNA1 showed better efficiency we chose to carry out the subsequent experiments with ANKHD1 shRNA1.

\subsection{ANKHD1 silencing arrest cells in S phase of cell cycle}

Cell cycle changes on inhibition of ANKHD1 was analyzed by flow cytometry. Separation of cells in G0/G1, S phase and G2/M was based on linear fluorescence intensity after staining with propium iodide. Cell cycle analysis showed significant percentage increase of cells in $S$ phase when MM cells were transduced with ANKHD1 shRNA (with not much difference to the proportion of G2 cells) as compared to controls, ${ }^{*} P<0.05$ (Fig. 5a). We also observed a concomitant decrease in the percentage of cells in G1 phase. Therefore ANKHD1 silencing caused accumulation of cells in the $S$ phase of the cycle.

\subsection{Analysis of genes relevant to cell cycle progression and proliferation}

To address the mechanism of the antiproliferative effect of ANKHD1 silencing, we examined the effect of ANKHD1 knockdown on the expression of cell cycle and proliferation related genes, including Cyclin dependent kinase inhibitors-p21 (CDKN1A) and p27 (CDKN1B), Cyclins-cyclin A2 (CCNA2), cyclin B1 (CCNB1) and cyclin E1 (CCNE1), Cyclin dependent kinases-CDK2 and CDK4 and Tumour suppressor protein p53 (TP53). Following the inhibition of ANKHD1 in U266, mRNA levels of CDKN1B, CCNB1and CCNE1 were reduced by $42.2 \%, 23.1 \%$ and $30 \%$ compared to control, respectively (Fig. 5b, above panel). Similarly on downregulation of ANKHD1 in MM1S cells, mRNA levels for CDKN1B, CCNB1 and CCNE1 were reduced by $42.3 \%, 23.2 \%$ and $18 \%$, respectively (Fig. 5b, below panel). On the other hand, CDKN1A was highly upregulated in both U266 ( $>100 \%$ ) and MM1S (52\%) cell lines on downregulation of ANKHD1 (Fig. 5b). No significant changes in CCNA2, CDK2 and CDK4 were observed on ANKHD1 silencing of both U266 and MM1S cells at mRNA levels.

At protein levels as determined by western blot, the two cell lines differed in levels of cyclin A protein with increase in U266 but a decrease in MM1S cells (Fig. 5c). However in contrast to qRT-PCR results where there was no significant difference in $C D K 2$ and $C D K 4$, there was a decrease in cdk2 and cdk4 at protein levels in both cell lines with almost complete inhibition in U266 cells (Fig. 5c). On the other hand in correlation with mRNA levels, the level of p27 protein was reduced and p21 expression increased in both cells with ANKHD1 silencing.

The two cell lines however showed difference in TP53 mRNA expressions being up regulated in MM1S (50\% increase), but down regulated in U266 (52\% decrease) cells with ANKHD1 inhibition (Fig. 5b). These results correlated well with p53 protein levels as determined by Western blot (Fig. 5c).

\section{Discussion}

ANKHD1 is reported to be highly expressed in leukemia cell lines and is suspected to have a role in cancer development [14]. We investigated the expression of ANKHD1 in MM and the effect of ANKHD1-targeted lentiviral shRNA on cell proliferation, apoptosis and cell cycle in MM cell lines U266 (glucocorticoid resistant) and MM1S (glucocorticoid sensitive) [16]. In this study, the expression of ANKHD1 in MM patients and cell lines was analyzed 
A

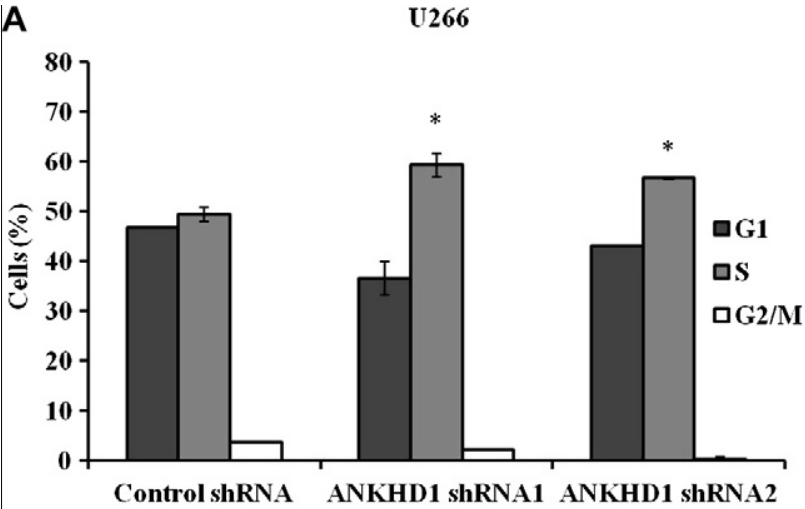

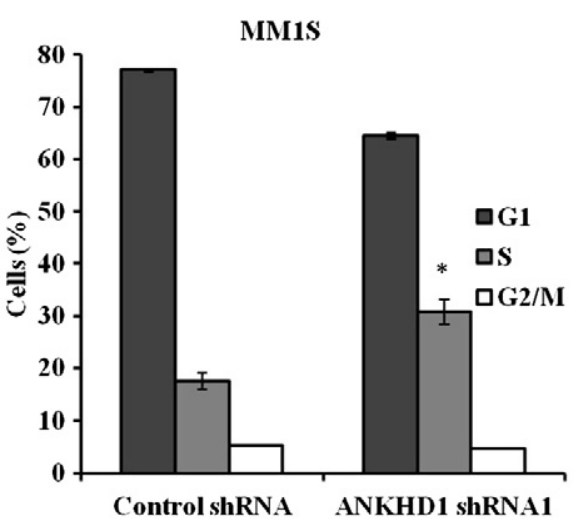

B

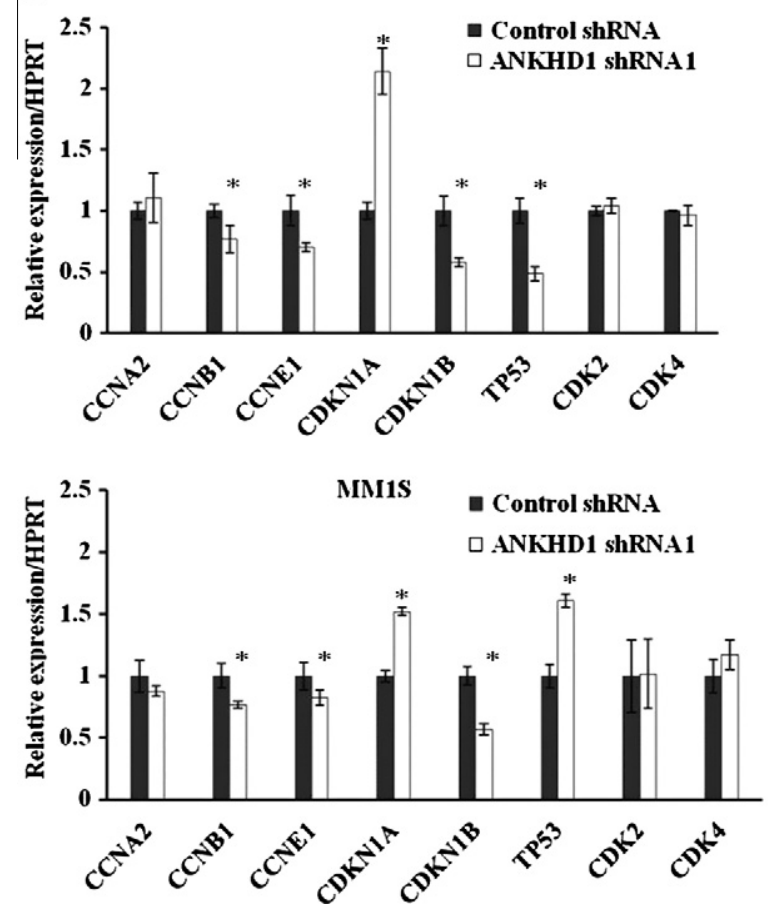

C
Control shRNA AXKHD1 shRNA1 Control shRVA ANKHD1 shRNAl

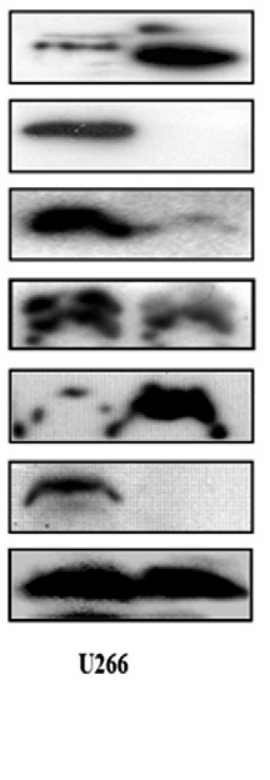

Cyclin A

Cdk4

Cdk2

p27

p21

p53

Actin
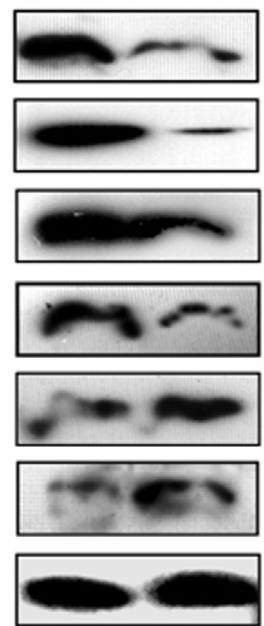

MM1S

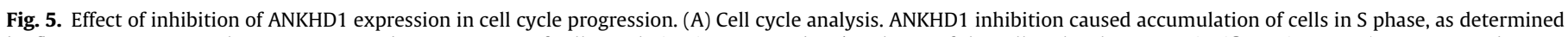

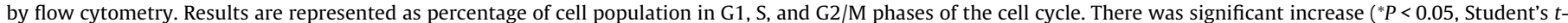

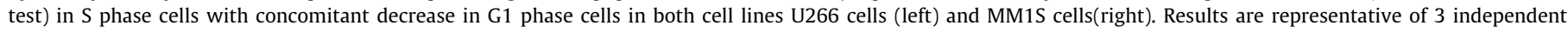

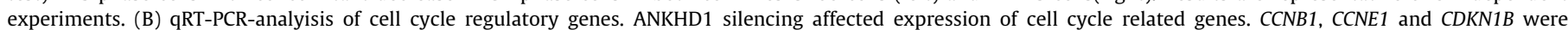

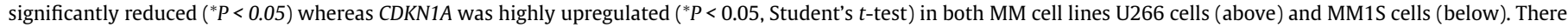

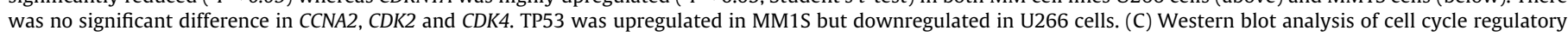

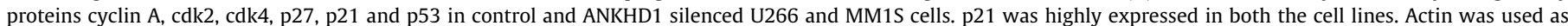
endogenous control.

by qRT-PCR. The results demonstrated that ANKHD1 was overexpressed in MM patients and cell lines. Although the number of patient samples studied were limited $(n=10)$, all of them showed increased expression of ANKHD1 mRNA as compared to negative controls. Furthermore, qRT-PCR results were corroborated with western blot analysis that confirmed overexpression of ANKHD1 in MM cell lines.

ANKHD1 overexpression suggests a potential role of this protein in the MM pathophysiology. Previous studies with acute leukemia patients and cell lines showed that ANKHD1 is associated with SHP2, which had a possible role in cell proliferation and apoptosis [14]. In the present study we sought to elucidate ANKHD1 role in proliferation and apoptosis by inhibiting its expression in glucocorticoid resistant U266 and sensitive MM1S cell lines. We used lentiviral vector delivering shRNA specific to human ANKHD1 gene.
One major problem of using shRNAs in experimentation is the possibility of off-target effects. Hence to confirm the specificity of RNAi results, we used two shRNAs targeting two different regions on ANKHD1 gene. In addition we used shRNA targeting LacZ gene (irrelevant gene) as negative control.Both the shRNAs used were able to knockdown ANKHD1 expression significantly $\left({ }^{*} P<0.05\right)$ at both mRNA and protein levels. Similar results were obtained on proliferation, apoptosis and cell cycle analysis with both shRNAs. However transduction efficiency and effects of knockdown of ANKHD1 expression was higher in cells transduced with ANKHD1 shRNA1 and therefore ANKHD1 shRNA1 was chosen for subsequent use in other myeloma cell line MM1S. Downregulation of ANKHD1 expression was validated by qRT-PCR and western blot following lentiviral transduction and selection and was significantly downregulated at both mRNA and protein levels in both cell 
lines used $\left({ }^{*} P<0.05\right)$. MTT assay was used to evaluate cell proliferation and revealed a significant decrease in proliferation of cells transduced with lentiviral ANKHD1 shRNA compared to control shRNA $\left({ }^{*} P<0.05\right)$ in both U266 and MM1S cell lines.

Following the downregulation of ANKHD1, cell growth was inhibited, whereas there was no induction of cell apoptosis. Changes in cell proliferation are usually associated with modulation in the cell cycle $[17,18]$. Hence in order to explore the potential mechanism underlying the action of ANKHD1 expression in regulating cell proliferation, cell cycles were characterized by FACS analysis. We observed significant increase in $S$ phase cells after transduction with lentiviral ANKHD1 shRNA as compared to cells tranduced with control shRNA. There was a concomitant decrease in G1 phase cells with no significant changes in the percentage of G2M phase cells. These findings suggest a role of ANKHD1 in cell cycle progression and probably, the delay in progression from $S$ to $\mathrm{G} 2 / \mathrm{M}$ or arrest in $\mathrm{S}$ phase was the reason for the antiproliferative effect of ANKHD1 suppression in myeloma cells. Having observed the accumulation of cells in S phase in ANKHD1 silenced cells, we hypothesized that ANKHD1 downregulation might change the expression of cell cycle genes. We examined by qRT-PCR analysis, the levels of expression of several cell cycle related genes, to further characterize the effect of ANKHD1 downregulation. We found that expression of CDKN1B (p27), a negative regulator of cell cycle and inhibitor of G1 phase cyclin/cdk activity [19,20], was downregulated on ANKHD1 inhibition. Hence, the progression from G1 to $S$ was not inhibited and there was a decrease in G1 phase cells. Contrarily, p21 (CDKN1A) that plays a major role in $\mathrm{S}$ phase progression was highly upregulated at both mRNA and protein levels, suggesting that the perturbation in $S$ phase progression was due to the negative regulation of cell cycle due to inhibition by p21 [21-23]. Furthermore, downregulation of ANKHD1 decreased, cyclin B and cyclin E, G1 phase and G2 phase proteins, whereas there was no significant change in cyclin A which is the S phase protein [24].

At protein levels, expression of cdk2, cdk4, p27 (CDKN1B) was decreased in both the cell lines with almost complete inhibition of expression in U266 cells. On the other hand there was an increase in cyclin A in U266 cells but a decrease in MM1S cells. Thus the effects of ANKHD1 downregulation on the expression of cyclin A appears to vary among cell systems. The observed decrease in cyclin A in MM1S cells was consistent with the observed delay in cell cycle progression as cyclin A regulates S/G2 progression but its upregulation in U266 could not be explained requiring further investigation. The probable reason however, for inhibition of cell cycle progression in U266 despite the high expression of cyclin A could be attributed to the decrease of cdk2 total protein. Cdk2 is cyclin dependent kinase that binds to cyclin A and whose activity is required for progression through $S$ phase $[25,26]$. In absence of cdk2, cyclin A alone cannot lead S to G2 progression. Decrease in cdk2 protein may be correlated to decrease in cyclin/cdk2 activity due to upregulation of p21 that regulates cell cycle progression, leading to delay in S phase progression [26,27].

We also analyzed expression of the tumour suppressor gene, TP53, which is a potent transcriptional regulator of p21 and found that TP53 was upregulated in MM1S but downregulated in U266 cells on downregulation of ANKHD1. The reason for this difference may be due to the fact that p53 is wild type in MM1S but mutated in U266 cell line [28]. Based on this finding, we can suggest that inhibition of ANKHD1 in myeloma cell growth is independent of p53 status of cells and the arrest of S phase might be mainly due to upregulation of p21, induced by ANKHD1 inhibition [29-31]. However, exactly how ANKHD1 inhibition leads to upregulation of p21 causing accumulation of cells in the $S$ phase is not yet clear and requires further investigation.

In conclusion, the present study demonstrates that ANKHD1 is highly expressed in both MM patients and cell lines. Further knockdown of ANKHD1 using shRNA potently inhibits proliferation and promotes cell cycle arrest without affecting rate of apoptosis in both glucocorticoid resistant as well as sensitive multiple myeloma cells. ANKHD1 overexpression may be associated with cell cycle progression via downregulation of p21, independent of p53 in MM cells. Further investigation of the functional role of ANKHD1 may lead to a better understanding of the molecular mechanism of multiple myeloma. Also, studies with combination of drugs that induce apoptosis and suppression of ANKHD1 may be an effective strategy for treatment of myeloma, and therefore needed to be explored.

\section{Acknowledgments}

The authors wish to thank Dr. Luis G.R. Fernandes, Tereza Salles, Raquel S. Foglio and Simone Sene for assistance. Authors also thank Dr. Rashmi Saini for helping in confocal microscopy. This work was supported by Instituto Nacional de Ciência e Tecnologia do Sangue(INCTS) and Conselho Nacional de Desenvolvimento Científico e Tecnológico (CNPq), Brazil.

\section{Appendix A. Supplementary data}

Supplementary data (effect of inhibition of ANKHD1 expression on MM cell proliferation. Proliferation was determined by MTT assay at 24,48 and $72 \mathrm{~h}$ of culture, post-seeding. Significant $\left({ }^{*} P<0.05\right.$, Student's $t$-test) inhibition was observed at all three time points with highest inhibition at $48 \mathrm{~h}$ ) associated with this article can be found, in the online version, at http://dx.doi.org/ 10.1016/j.febslet.2012.10.037.

\section{References}

[1] Lopez-Corral, L., Gutierrez, N.C., Vidriales, M.B., Mateos, M.V., Rasillo, A., Garcia-Sanz, R., Paiva, B. and San Miguel, J.F. (2011) The progression from MGUS to smoldering myeloma and eventually to multiple myeloma involves a clonal expansion of genetically abnormal plasma cells. Clin. Cancer Res. 17, 1692-1700.

[2] Palumbo, A. and Anderson, K. (2011) Multiple myeloma. N. Engl. J. Med. 364, 1046-1060.

[3] Raab, M.S., Podar, K., Breitkreutz, I., Richardson, P.G. and Anderson, K.C. (2009) Multiple myeloma. Lancet 374, 324-339.

[4] Bergsagel, P.L. and Kuehl, W.M. (2001) Chromosome translocations in multiple myeloma. Oncogene 20, 5611-5622.

[5] Joy Ho, P. (2002) Chromosomal and genetic abnormalities in myeloma. Clin. Lab. Haematol. 24, 259-269.

[6] Merchionne, F., Perosa, F. and Dammacco, F. (2007) New therapies in multiple myeloma. Clin. Exp. Med. 7, 83-97.

[7] Kumar, S.K. et al. (2008) Improved survival in multiple myeloma and the impact of novel therapies. Blood 111, 2516-2520.

[8] Child, J.A. et al. (2003) High-dose chemotherapy with hematopoietic stem-cell rescue for multiple myeloma. N. Engl. J. Med. 348, 1875-1883.

[9] Mesri, M., Wall, N.R., Li, J., Kim, R.W. and Altieri, D.C. (2001) Cancer gene therapy using a surviving mutant adenovirus. J. Clin. Invest. 108, 981-990.

[10] Semenza, G.L. (2003) Targeting HIF-1 for cancer therapy. Nat. Rev. Cancer 3 , $721-732$

[11] Weber, G.F. (2001) The metastasis gene osteopontin: a candidate target for cancer therapy. Biochim. Biophys. Acta 1552, 61-85.

[12] Chen, L., Liu, Q., Qin, R., Le, H., Xia, R., Li, W. and Kumar, M. (2005) Amplification and functional characterization of MUC1 promoter and genevirotherapy via a targeting adenoviral vector expressing hSSTR2 gene in MUC1-positive Panc-1 pancreatic cancer cells in vitro. Int. J. Mol. Med. 15, 617-626.

[13] Boehm, J.S. and Hahn, W.C. (2011) Towards systematic functional characterization of cancer genomes. Nat. Rev. Genet. 12, 487-498.

[14] Traina, F., Favaro, P.M., Medina Sde, S., Duarte Ada, S., Winnischofer, S.M., Costa, F.F. and Saad, S.T. (2006) ANKHD1, ankyrin repeat and KH domain containing 1, is overexpressed in acute leukemias and is associated with SHP2 in K562 cells. Biochim. Biophys. Acta 1762, 828-834.

[15] Machado-Neto, J.A., Favaro, P., Lazarini, M., Costa, F.F., Olalla Saad, S.T. and Traina, F. (2011) Knockdown of insulin receptor substrate 1 reduces proliferation and downregulates Akt/mTOR and MAPK pathways in K562 cells. Biochim. Biophys. Acta 1813, 1404-1411.

[16] Rizvi, M.A., Ghias, K., Davies, K.M., Ma, C., Weinberg, F., Munshi, H.G., Krett, N.L. and Rosen, S.T. (2006) Enzastaurin (LY317615), a protein kinase Cbeta inhibitor, inhibits the AKT pathway and induces apoptosis in multiple myeloma cell lines. Mol. Cancer Ther. 5, 1783-1789. 
[17] Stiller, D. and Katenkamp, D. (1978) Intercellular substances in Hodgkin's lymphomas. Ultrastructural investigations. Virchows Arch. A Pathol. Anat. Histol. 380, 81-90

[18] Belanger, S., Cote, M., Lane, D., L'Esperance, S., Rancourt, C. and Piche, A. (2005) Bcl-2 decreases cell proliferation and promotes accumulation of cells in S phase without affecting the rate of apoptosis in human ovarian carcinoma cells. Gynecol. Oncol. 97, 796-806.

[19] Haferlach, C., Bacher, U., Kohlmann, A., Schindela, S., Alpermann, T., Kern, W., Schnittger, S. and Haferlach, T. (2011) CDKN1B, encoding the cyclindependent kinase inhibitor 1B (p27), is located in the minimally deleted region of $12 \mathrm{p}$ abnormalities in myeloid malignancies and its low expression is a favorable prognostic marker in acute myeloid leukemia. Haematologica 96, 829-836.

[20] Molatore, S., Kiermaier, E., Jung, C.B., Lee, M., Pulz, E., Hofler, H., Atkinson, M.J. and Pellegata, N.S. (2010) Characterization of a naturally-occurring p27 mutation predisposing to multiple endocrine tumors. Mol. Cancer Ther. 9, 116.

[21] Liu, J., Hu, J.L., Shi, B.W., He, Y. and Hu, W.X. (2010) Up-regulation of p21 and TNF-alpha is mediated in lycorine-induced death of HL-60 cells. Cancer Cell Int. 10, 25.

[22] Kuo, P.L., Ni, W.C., Tsai, E.M. and Hsu, Y.L. (2009) Dehydrocostuslactone disrupts signal transducers and activators of transcription 3 through upregulation of suppressor of cytokine signaling in breast cancer cells. Mol. Cancer Ther. 8, 1328-1339.

[23] Gottifredi, V., McKinney, K., Poyurovsky, M.V. and Prives, C. (2004) Decreased p21 levels are required for efficient restart of DNA synthesis after S phase block. J. Biol. Chem. 279, 5802-5810.
[24] Johnson, D.G. and Walker, C.L. (1999) Cyclins and cell cycle checkpoints. Annu. Rev. Pharmacol. Toxicol. 39, 295-312.

[25] Woo, R.A. and Poon, R.Y. (2003) Cyclin-dependent kinases and S phase control in mammalian cells. Cell Cycle 2, 316-324.

[26] Peiretti, A., Baghdassarian, N., Gerland, L.M., Ffrench, P., Bryon, P.A., Magaud, J.P. and Ffrench, M. (2003) CDK2 is involved in the S-phase lengthening induced by glucocorticoids in normal human lymphocytes. Eur. J. Cell Biol. 82 253-261.

[27] Zhu, Y., Alvarez, C., Doll, R., Kurata, H., Schebye, X.M., Parry, D. and Lees, E. (2004) Intra-S-phase checkpoint activation by direct CDK2 inhibition. Mol. Cell. Biol. 24, 6268-6277.

[28] Saha, M.N., Jiang, H., Mukai, A. and Chang, H. (2010) RITA inhibits multiple myeloma cell growth through induction of p53-mediated caspase-dependent apoptosis and synergistically enhances nutlin-induced cytotoxic responses. Mol. Cancer Ther. 9, 3041-3051.

[29] Yang, X., Wang, W., Qin, J.J., Wang, M.H., Sharma, H., Buolamwini, J.K., Wang H. and Zhang, R. (2012) JKA97, a novel benzylidene analog of harmine, exerts anti-cancer effects by inducing G1 arrest, apoptosis, and p53-independent upregulation of p21. PLoS One 7, e34303.

[30] Takagaki, N., Sowa, Y., Oki, T., Nakanishi, R., Yogosawa, S. and Sakai, T. (2005) Apigenin induces cell cycle arrest and p21/WAF1 expression in a p53independent pathway. Int. J. Oncol. 26, 185-189.

[31] Lin, K., Rockliffe, N., Johnson, G.G., Sherrington, P.D. and Pettitt, A.R. (2008) Hsp90 inhibition has opposing effects on wild-type and mutant p53 and induces p21 expression and cytotoxicity irrespective of p53/ATM status in chronic lymphocytic leukaemia cells. Oncogene 27, 2445-2455. 